\title{
Distributed Cooperative Current-Sharing Control of Parallel Chargers Using Feedback Linearization
}

\author{
Jiangang Liu, ${ }^{1}$ Zhiwu Huang, ${ }^{1}$ Jing Wang, ${ }^{1,2}$ Jun Peng, ${ }^{1}$ and Weirong Liu' ${ }^{1}$ \\ ${ }^{1}$ Hunan Engineering Laboratory for Advanced Control and Intelligent Automation, School of Information Science and Engineering, \\ Central South University, Changsha, Hunan 410075, China \\ ${ }^{2}$ School of Science, Engineering and Mathematics, Bethune-Cookman University, Daytona Beach, FL 32114, USA
}

Correspondence should be addressed to Zhiwu Huang; hzw@csu.edu.cn

Received 16 December 2013; Accepted 19 February 2014; Published 7 April 2014

Academic Editor: Guoqiang Hu

Copyright (C) 2014 Jiangang Liu et al. This is an open access article distributed under the Creative Commons Attribution License, which permits unrestricted use, distribution, and reproduction in any medium, provided the original work is properly cited.

\begin{abstract}
We propose a distributed current-sharing scheme to address the output current imbalance problem for the parallel chargers in the energy storage type light rail vehicle system. By treating the parallel chargers as a group of agents with output information sharing through communication network, the current-sharing control problem is recast as the consensus tracking problem of multiagents. To facilitate the design, input-output feedback linearization is first applied to transform the nonidentical nonlinear charging system model into the first-order integrator. Then, a general saturation function is introduced to design the cooperative current-sharing control law which can guarantee the boundedness of the proposed control. The cooperative stability of the closed-loop system under fixed and dynamic communication topologies is rigorously proved with the aid of Lyapunov function and LaSalle invariant principle. Simulation using a multicharging test system further illustrates that the output currents of parallel chargers are balanced using the proposed control.
\end{abstract}

\section{Introduction}

As a new type of electric traction light rail transportation system, the energy storage type light rail vehicle, adopts supercapacitors as its power supply. With this energy storage technology, there is no need to construct a traction power grid and it is possible to recover the energy of regenerative braking. Energy storage type light rail vehicles need to be charged in seconds by the charging system when parking at the platform. Therefore, the charging system should provide a large enough output power to shorten the charging process. An effective method to solve this problem would be by connecting several chargers in parallel to increase system capacity [1], as shown in Figure 1. However, the challenge for the charging system is how to balance the charging current between the chargers.

If the charging current is not balanced, the charger with a higher output current has to bear a greater output power, which may lead to a large thermal stress and degrade the reliability and performance of the whole charging system.
It is therefore necessary to design an effective current-sharing control strategy to balance the output current for charging system.

There are several conventional approaches for currentsharing problems, such as central current-sharing control method, droop control method $[2,3]$, and master-slave method $[4,5]$. The central current-sharing control method needs a central controller to compute each module's output current according to the total load current. The central controller sends a unified command to balance the current. This method is relatively simple and easy to implement. However, if the central controller is faulty, this may result in the collapse of the whole charging system and degrade the whole system's reliability.

Under the droop control method, the output voltage decreases as the output current increases, effectively controlling and changing the output impedance to achieve balance $[2,3]$. The method is simple and has been widely used in small-capacity parallel charging systems. However, this approach is not suitable for large-power paralleled charging 


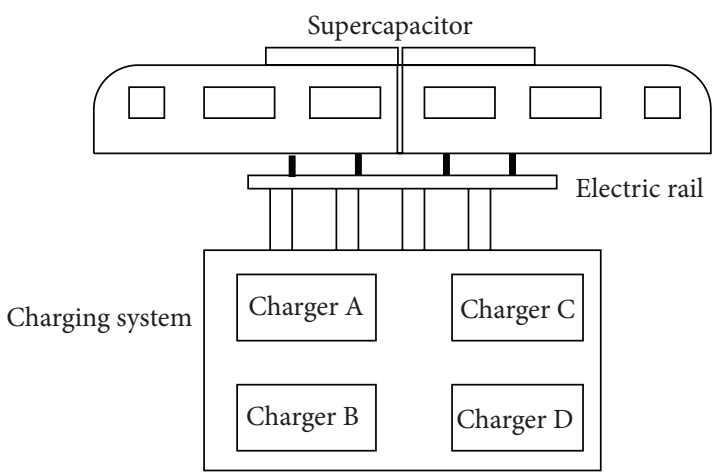

FIGURE 1: The schematic diagram of charging system for the energy storage type light rail vehicle system.

system and is not effective when the load has relatively large changes.

Master-slave current-sharing control is manually specifying one module among $N$ parallel power modules as the main module and the remaining module's current follows the main module's allocation current $[4,5]$. This method is suitable for a double closed-loop control system with an external voltage loop and an inner current loop. However, this method has the same drawback as the central current-sharing control approach, which means that if the main module is faulty, the whole system will collapse.

Central current-sharing control method, droop control method, and master-slave method are undistributed and restrictive and have some drawbacks and limitations, which are not the appropriate approach for the charging system of energy storage type light rail vehicles when the chargers are parallel with each other. Taking distributed chargers in the charging system as agents in multiagent systems, the currentsharing control design resembles a current consensustracking problem, where the charger's current tracks the reference. Several chargers are connected in parallel with each other in the charging system of an energy storage type light rail vehicle. The adjacent chargers interact with each other and exchange the state information such as the charging current. This method is fully distributed, each charger only requires its own information and the information of some neighbors, and one faulty node cannot cause the collapse of the whole charging system, which is an appropriate method for the charging system to solve the current-sharing problem.

Over the last decades, consensus and cooperative control of multiagent systems have received most of the attention [6], giving results in the areas such as flocking [7], formation control [8], distributed mobile sensor network [9], rendezvous in space, and autonomous vehicles [10]. Distributed consensus and cooperative control have also been recently introduced in power systems to regulate the output power of multiple photovoltaic generators [11], which has also been used to synchronize the output voltage of distributed generators in microgrids [12]. Although the cooperative control has been applied in power system, the control input constraints were not considered in detail.
However, the charging system has some physical constraints, such as the duty ratio. And there are inevitably components error and manufacturing error in each charger system; the dynamics of the chargers in the charging system are nonidentical. The charger has intrinsic nonlinear characteristics because of Buck DC/DC circuit's principle and super capacitor's feature. This paper seeks to address the challenge of how to design the cooperative current-sharing control law and take into account both the charger's nonidentical and nonlinear features.

The main contribution of this paper can be summarized as follows.

The current-sharing control of charging system for energy storage type light rail vehicles is implemented through the concept of distributed cooperative control of multiagent systems. The current-sharing problem is converted into the consensus-tracking problem of nonlinear and nonidentical multiagent systems.

Input-output feedback linearization is introduced to transform the nonidentical dynamics into first-order integrator. A general saturation function is introduced to design the cooperative current-sharing control law based on neighborhood rule. The Lyapunov function integrating LaSalle invariant principle is adopted to prove the cooperative stability of closed-loop systems.

The proposed current-sharing control scheme is implemented through a sparse communication network, which is essentially distributed. The communication network is modeled by a graph. Each charger only requires its own current information and that of its neighbors.

The rest of this paper is organized as follows: currentsharing problems of the charging system are set out in Section 2, the current-sharing control strategy based on distributed cooperative control of multiagent systems is proposed in Section 3, and cooperative stability is proved in Section 4 . The proposed current-sharing strategy is verified in Section 5. Section 6 concludes this paper.

\section{Charging System Problem Formulation}

Considering the charging system for the energy storage type light rail vehicle, as shown in Figure 2, the charging system consists of a $10 \mathrm{kV}$ AC supply grid, a $10 \mathrm{kV} / 900 \mathrm{~V}$ AC converter, and a network of parallel charger subsystems. All of the charger subsystems are able to exchange the state information through communications.

Each charger subsystem is composed of a three-phase bridge rectifier circuit and a chopper BUCK DC/DC circuit (Figure 3). The current dynamics in the BUCK circuit can be established based on Kirchhoff's Voltage Law. To this end, when the IGBT (Insulated Gate Bipolar Transistor) is ON, the dynamics of the current in the BUCK circuit are described as

$$
\dot{I}_{i}=\frac{U_{d_{i}}}{L_{i}} D_{i}-\frac{I_{i} r_{i}}{L_{i}}-\frac{U_{c}}{L_{i}},
$$

and when the IGBT is OFF, we have

$$
\dot{I}_{i}=-\frac{I_{i} r_{i}}{L_{i}}-\frac{U_{c}}{L_{i}}
$$




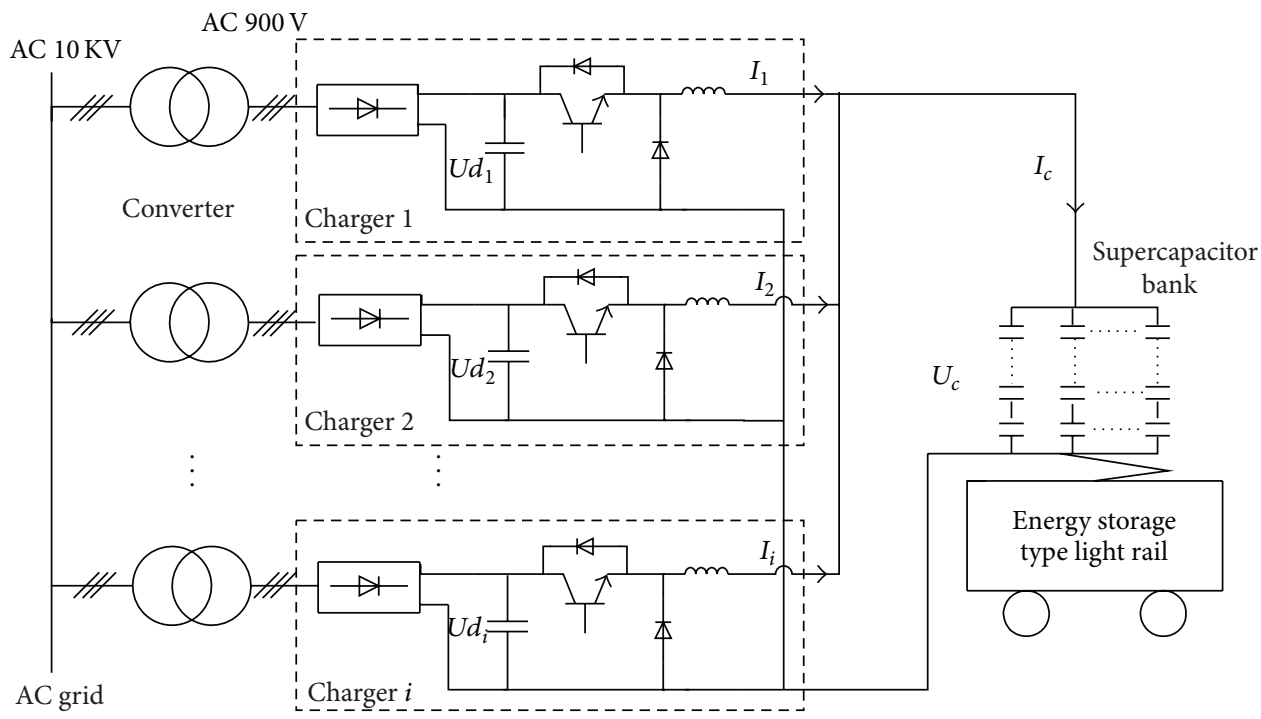

FIgURE 2: The main circuit schematic diagram of charging system for energy storage type light rail.

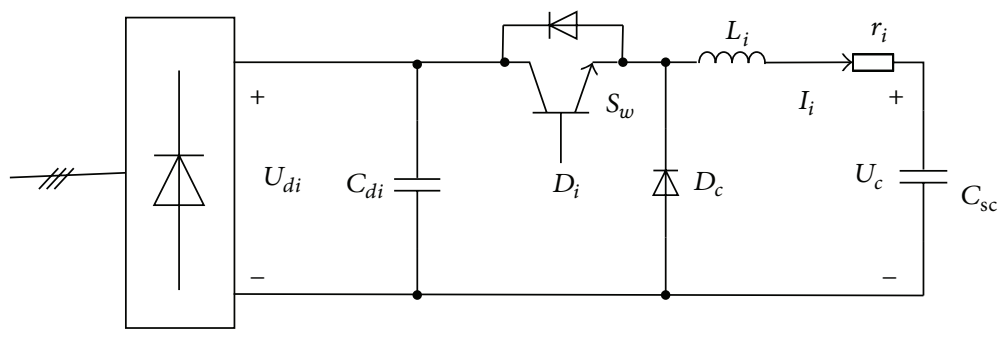

FIGURE 3: Buck circuit for each charger subsystem.

where $I_{i}$ is the output current of the $i$ th charging subsystem, $U_{c}$ is supercapacitor's voltage, $U_{d_{i}}$ is the DC input voltage which is obtained from the three-phase bridge rectifier, $D_{i}$ is the IGBT duty ratio, $L_{i}$ is the flux for the energy storage inductor, and $r_{i}$ is the equivalent resistance of the circuit.

It can be seen from Figure 3 that supercapacitor is the key element in the energy storage light rail vehicle system. We use $C_{\mathrm{sc}}$ to denote its capacitance, and its value may ascend as the voltage increases. There exists electric charge redistribution process at the end of the charging stage, and a leakage current exists as well. Such features can be described by their equivalent three-branch RC circuit shown in Figure 4. The first branch is the instantaneous branch, which includes a resistor $R_{1}$, two capacitors $C_{0}$ and $C_{v}$. Voltage-dependent capacitance $C_{v}$ relies on the terminal voltage $U_{c}$. The second branch is the delay branch, which includes a resistor $R_{2}$ and a capacitor $C_{2}$. The third branch is the long term branch, which includes a resistor $R_{3}$ and a capacitor $C_{3}$. $R_{\text {lea }}$ is the leakage resistor. The time constants for the instantaneous branch, the delay branch, and the long term branch are different, and the values are in the level of seconds, minutes, and ten minutes, respectively. The charging process is mainly dependent on the first RC branch with the smallest time constant which meets the fast charging requirement for the energy storage type light rail vehicle system.
The relationship between capacitance and voltage for the super capacitor is given by

$$
C_{\mathrm{sc}}=C_{0}+C_{v} U_{c} .
$$

The voltage dynamic can be described by the following nonlinear differential equation:

$$
\begin{aligned}
\dot{U}_{c} & =\frac{I_{c}}{C_{\mathrm{sc}}} \\
& =\frac{I_{c}}{C_{0}+C_{v} U_{c}} \\
& =\frac{1}{C_{0}+C_{v} U_{c}} \sum_{i=1}^{n} I_{i},
\end{aligned}
$$

where $I_{c}$ is the total charging current, $I_{i}$ is the output current of the $i$ th charging subsystem, and $n$ is the number of the total charging subsystems.

To this end, it follows from (1)-(4) that the system model for the $i$ th charging subsystem can be described as

$$
\begin{aligned}
\dot{x}_{i} & =f_{i}\left(x_{1}, x_{2}, \ldots, x_{n}\right)+g_{i}\left(x_{i}\right) u_{i}, \\
y_{i} & =h_{i}\left(x_{i}\right),
\end{aligned}
$$




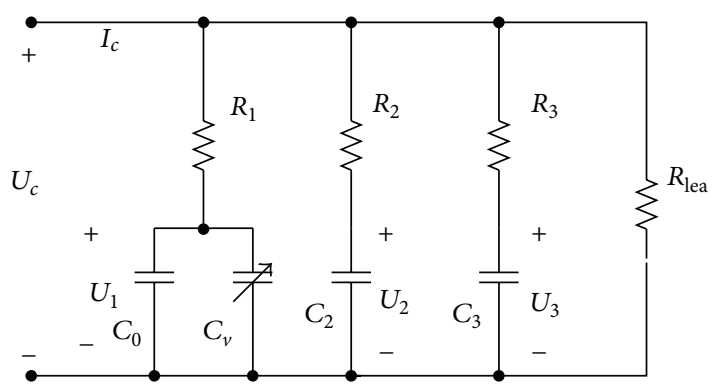

FIgURE 4: Equivalent three branches RC circuit model of the supercapacitor.

where $i=1,2, \ldots, n, x_{i}=\left[I_{i}, U_{c}\right]^{T} \in R^{2}$ is ith charger's state, $u_{i}(t)=U_{d_{i}} D_{i} \in R$ is the control input, and system functions $f_{i}\left(x_{1}, x_{2}, \ldots, x_{n}\right)$ and $g_{i}\left(x_{i}\right)$ are Lipschitz continuous and defined below

$$
\begin{aligned}
f_{i}\left(x_{1}, x_{2}, \ldots, x_{n}\right) & =\left[\begin{array}{cc}
-\frac{r_{i}}{L_{i}} & -\frac{U_{c}}{L_{i}} \\
\frac{1}{C_{0}+C_{v} U_{c}} & \sum_{k=1}^{n} I_{k}
\end{array}\right], \\
g_{i}\left(x_{i}\right) & =\left[\begin{array}{c}
\frac{1}{L_{i}} \\
0
\end{array}\right], \\
h_{i}\left(x_{i}\right) & =I_{i} .
\end{aligned}
$$

For the charging system of the energy storage light rail vehicle, there are several modeling methods. In this paper, the average value modeling method is adopted to describe the dynamic of the charger. The current $I_{i}$ and voltage $U_{c}$ are chosen as the charger's state $x_{i} . f_{i}\left(x_{1}, x_{2}, \ldots, x_{n}\right), g_{i}\left(x_{i}\right)$ are the nonlinear functions, which describe the nonlinear and nonidentical charger and are related with the current $I_{1}, I_{2}, \ldots, I_{n}$ and voltage $U_{c}$. When we choose average value modeling method, the voltage $U_{d_{i}} D_{i}$ is chosen as the control input $u_{i}$, which is bounded because the duty ratio is within a range. The current $I_{i}$ is considered as the output of the charger; that is, $y_{i}=h_{i}\left(x_{i}\right)=I_{i}$.

To ensure the charging performance for the energy storage rail vehicle system, it is expected that the output current $I_{i}$ for all charging subsystems would be identical. However, in practice, it is unavoidable that the DC input voltages $U_{d_{i}}$ for the charging subsystems may not be same, which results in the unbalanced output current $I_{i}$. To solve this problem, in this paper, the control objective is to design a cooperative control $u_{i}(t)$ such that

$$
\lim _{t \rightarrow+\infty}\left|y_{i}(t)-y_{0}\right|=0
$$

where $y_{0}$ is the reference current.

The reference current $y_{0}$ is a prespecified parameter. In this paper, we assume that this information is only available to one of the charging subsystems. Through information exchanges among the charging subsystems, the proposed cooperative control is designed to achieve the control objective defined in (7).
In real applications, $y_{0}$ is calculated by $y_{0}=I_{c} / n$, given $n$ parallel subsystems in the current charging process, where $I_{c}$ represents the total charging current. Once the desired voltage $U_{c}\left(t_{d}\right)$ within the required charging time $t_{d}$, the initial capacitances $C_{\mathrm{sc}}(0)$, and the initial residual voltage $U_{c}(0)$ of supercapacitors are given, $I_{c}$ can be computed by $I_{c}=$ $C_{\mathrm{sc}}(0)\left(U_{c}\left(t_{d}\right)-U_{c}(0)\right) / t_{d}$ in advance.

Each controller communicates with each other through RS-422 bus. And every controller can know whether other controllers are involved in the charging process or not. Therefore, the number $n$ can be counted. When some charger cannot work, the desired setpoint reference current $y_{0}$ would be different accordingly as the total number $n$ of working charging subsystems during that stage has changed. But $y_{0}$ cannot exceed the maximum current $I_{\max }$ that each charger can afford.

The cooperative current-sharing objective $y_{0}$ indeed depends on the number $n$ and the total charging current $I_{c}$. It is worth mentioning that the messages about $y_{0}$ are only required to be configured to one of charging subsystems in the network. And due to the nature of the proposed cooperative control, all other subsystems in the network will eventually converge to the newly updated setpoint $y_{0}$.

\section{Current-Sharing Strategy Based on Distributed Cooperative Control}

To design the distributed cooperative current-sharing control for the charging system, we assume that the charging subsystems are able to exchange information through communication network. We use a graph to describe such a communication network. In this section, after some preliminaries on graph theory, we present the proposed cooperative control for distributed current-sharing control based on input-output feedback linearization technique.

3.1. Preliminary of Graph Theory. The communication topology of a multiagent system can be modeled by a graph [13]. A graph is usually denoted as $G(\nu, \varepsilon, A)$ with a nonempty finite set of $n$ nodes $v=\left\{v_{1}, v_{2}, \ldots, v_{n}\right\}$, a set of edges or arcs $\varepsilon \subseteq$ $\nu \times \nu$, and the associated adjacency matrix $A=\left[a_{i j}\right] \in R^{n \times n}$.

When the communication topology is fixed, the adjacency matrix $A$ is constant; otherwise $A$ is time-varying; that is, $A(t)=\left[a_{i j}(t)\right]_{n \times n}$. An edge from node $j$ to node $i$ is denoted by $\left(v_{j}, v_{i}\right)$, which means that node $i$ receives the information from node $j . a_{i j}$ is the weight of edge $\left(v_{j}, v_{i}\right)$, and $a_{i j}>0$ if $\left(v_{j}, v_{i}\right) \in \varepsilon$; otherwise $a_{i j}=0$. The graph is assumed to have no self-loops, which means $a_{i i}=0$.

Node $j$ is called a neighbor of node $i$ if $\left(v_{j}, v_{i}\right) \in \varepsilon$. The set of neighbors of node $i$ is denoted as $N_{i}=\left\{v_{j} \in \nu:\left(v_{j}, v_{i}\right) \in \varepsilon\right\}$.

Definition 1. A graph without loops is called simple graph [7], that is, the adjacency matrix diagonal elements $a_{i i}=0$, and its Laplacian matrix is denoted as $L=\left[l_{i j}\right]_{n \times n}$ :

$$
l_{i j}= \begin{cases}\sum_{k=1, k \neq i}^{n} a_{i k}, & j=i \\ -a_{i j}, & j \neq i .\end{cases}
$$


The in-degree and out-degree of node $v_{i}$ are, respectively, defined as follows:

$$
\operatorname{deg}_{\text {in }}\left(v_{i}\right)=\sum_{j \in N_{i}} a_{i j}, \quad \operatorname{deg}_{\text {out }}\left(v_{i}\right)=\sum_{j \in N_{i}} a_{j i} .
$$

Definition 2 (balanced graphs). The node $i$ of a digraph $G(\nu, \varepsilon, A)$ is balanced if and only if its in-degree and outdegree are equal; that is, $\operatorname{deg}_{\text {in }}\left(v_{i}\right)=\operatorname{deg}_{\text {out }}\left(v_{i}\right)$. A graph $G(\nu, \varepsilon, A)$ is called balanced if and only if all of its nodes are balanced.

The in-degree matrix is defined as $D=\operatorname{diag}\left\{d_{i}\right\} \in R^{n \times n}$ with $d_{i}=\sum_{j \in N_{i}} a_{i j}$. The Laplacian matrix can also be defined as $L=D-A$. All row sums of $L$ are equal to zero; that is $L 1_{n}=0$, where $1_{n}$ is a column vector with $n$ elements equal to 1 .

A directed path from node $i$ to node $j$ is a sequence of edges, expressed as

$$
\left\{\left(v_{i}, v_{k}\right),\left(v_{k}, v_{l}\right), \ldots,\left(v_{m}, v_{j}\right)\right\} .
$$

A digraph is said to have a spanning tree, if there is a node $i_{r}$ (called the root), such that there is a directed path from the root to every other node in the graph.

Remark 3. When the communication topology is dynamic, the associated adjacency matrix is $A(t)=\left[a_{i j}(t)\right]_{n \times n}$; the Laplacian matrix is denoted as $L(t)=\left[l_{i j}(t)\right]_{n \times n}$, where

$$
l_{i j}(t)= \begin{cases}\sum_{k=1, k \neq i}^{n} a_{i k}(t), & j=i \\ -a_{i j}(t), & j \neq i .\end{cases}
$$

3.2. Current-Sharing Controller Design under Fixed Topology. To facilitate the cooperative design, we first convert the nonlinear model in (5) into the linear one through input-output feedback linearization. It follows that the time derivative of the output $y_{i}$ is

$$
\begin{aligned}
\dot{y}_{i} & =\frac{\partial h_{i}\left(x_{i}\right)}{\partial x_{i}} \dot{x}_{i} \\
& =\frac{\partial h_{i}\left(x_{i}\right)}{\partial x_{i}}\left(f_{i}\left(x_{1}, x_{2}, \ldots, x_{n}\right)+g_{i}\left(x_{i}\right) u_{i}\right) \\
& =L_{f_{i}} h_{i}+L_{g_{i}} h_{i} u_{i},
\end{aligned}
$$

where $L_{f_{i}} h_{i}$ is the Lie derivative of $h_{i}$ with respect to $f_{i}$ and is defined by $L_{f_{i}} h_{i}=\nabla h_{i} f_{i}=\left(\partial h_{i}\left(x_{i}\right) / \partial x_{i}\right) f_{i}\left(x_{1}, x_{2}, \ldots, x_{n}\right)$ and $L_{g_{i}} h_{i}$ is the Lie derivative of $h_{i}$ with respect to $g_{i}$ and is defined by $L_{g_{i}} h_{i}=\nabla h_{i} g_{i}=\left(\partial h_{i}\left(x_{i}\right) / \partial x_{i}\right) g_{i}\left(x_{i}\right)$. Define an auxiliary control $\vartheta_{i}$ as follows:

$$
\vartheta_{i}=L_{f_{i}} h_{i}+L_{g_{i}} h_{i} u_{i}
$$

Substituting (13) into (12), we have the first-order integrator model

$$
\dot{y}_{i}=\vartheta_{i}
$$

Since the model of the charger is based on converter by using average value modeling method, the duty cycle $D_{i}$ of Insulated Gate Bipolar Transistor is bounded and the voltage $U_{d_{i}} \cdot D_{i}$ is also bounded. Therefore, we introduce a saturation function $\phi(\cdot)$ in the proposed cooperative control to guarantee the boundedness of the control input.

Assumption 4. A general saturation function $\phi(\cdot)$ satisfies the following:
(1) $\phi(\cdot)$ is Lipschitz continuous,
(2) $\phi(z)=0 \Leftrightarrow z=0$,
(3) $z \phi(z)>0, \forall z \neq 0$,
(4) $\phi_{\min } \leq \phi(z) \leq \phi_{\max }, \forall z \in R$.

Remark 5. One possible choice of saturation functions is hyperbolic tangent function as used in [14]. Nonetheless, any function satisfying Assumption 4 would work. To this end, once $\vartheta_{i}$ is bounded, the original control input $u_{i}$ is also bounded based on inverse transformation of (13) since $f_{i}(\cdot)$ and $g_{i}(\cdot)$ are bounded. follows:

The proposed auxiliary control $\vartheta_{i}$ can be designed as

$$
\vartheta_{i}=c_{i}\left(\sum_{j \in N_{i}} a_{i j} \phi\left(y_{j}-y_{i}\right)+\rho_{i} \phi\left(y_{0}-y_{i}\right)\right),
$$

where $\phi(z)$ satisfy Assumption 4, $N_{i}=\left\{v_{j} \in \nu:\left(v_{i}, v_{j}\right) \in\right.$ $\varepsilon\}$ the set of neighbors of $v_{i}, a_{i j}>0$ means that for the $i$ th charger, it can receive the information of the $j$ th charger. $c_{i}>$ 0 is the coupling strength. The control parameter $c_{i}$ can be chosen appropriately to improve the response time [15]. $\rho_{i}$ is the pinning gain and $\rho_{i}>0$ for at least one $i$, which means that at least one charger knows the virtual charging current. From (15), we know that the current-sharing control law $\vartheta_{i}$ is only based on the $i$ th charger's current information and the information of its neighbors.

Remark 6. In order to make the current-sharing controller designing much more flexible for the nonidentical charging system, the different coupling strength $c_{i}$ is chosen in the updated cooperative control law for each subsystem instead of using the traditional common coupling strength $c$ [15]. The convergence speed can be improved by tuning $c_{i}$. In addition, $c_{i}$ does not depend on the whole network topology.

Accordingly, the cooperative control input $u_{i}$ can be carried out by $\vartheta_{i}$ as

$$
u_{i}=\frac{\vartheta_{i}-L_{f_{i}} h_{i}}{L_{g_{i}} h_{i}}
$$

When the communication topology is fixed, the final cooperative controller is formulated as follows by substituting (15) into (16):

$$
u_{i}=\frac{c_{i}\left(\sum_{j \in N_{i}} a_{i j} \phi\left(y_{j}-y_{i}\right)+\rho_{i} \phi\left(y_{0}-y_{i}\right)\right)-L_{f_{i}} h_{i}}{L_{g_{i}} h_{i}} .
$$




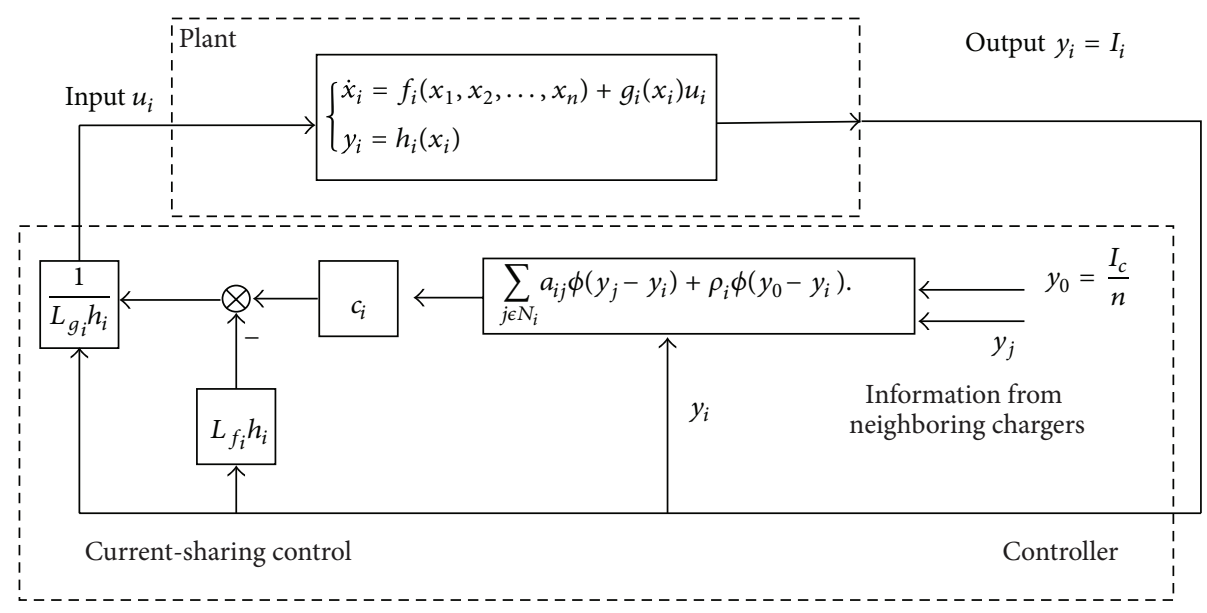

FIGURE 5: The block diagram of the proposed cooperative current-sharing control.

The block diagram of the proposed cooperative control is shown in Figure 5.

By substituting (17) into (12), we have each subsystem's closed-loop dynamics:

$$
\dot{y}_{i}=c_{i}\left(\sum_{j \in N_{i}} a_{i j} \phi\left(y_{j}-y_{i}\right)+\rho_{i} \phi\left(y_{0}-y_{i}\right)\right), \quad i=1,2, \ldots, n \text {. }
$$

As RS-422 full duplex communication protocol is used between each charger, each charger can communicate with each other in a bidirectional way. The communication topology is an undirected graph. Therefore, we analyze the undirected case in our work.

Assumption 7. The communication network between each chargers is bidirectional information flow, which means that the directed graph $G(\nu, \varepsilon, A)$ for the fixed communication topology is symmetric; that is, $a_{i j}=a_{j i}, i, j=1,2, \ldots, n$.

To facilitate the analysis in Section 4, let us further define the new auxiliary state variable $\delta_{i}(t)=y_{i}(t)-y_{0}$. Furthermore, each auxiliary subsystem's state equation can be written as

$$
\dot{\delta}_{i}=\dot{y}_{i}
$$

Furthermore, we can get

$$
\dot{\delta}_{i}=-c_{i}\left(\sum_{j \in N_{i}} a_{i j} \phi\left(\delta_{i}-\delta_{j}\right)+\rho_{i} \phi\left(\delta_{i}\right)\right), \quad i=1,2, \ldots, n
$$

3.3. Current-Sharing Controller Design under Dynamical Topology. When the communication topology is dynamic, the distributed cooperative controller is formulated as follows by substituting (15) into (16):

$$
u_{i}=\frac{c_{i}\left(\sum_{j \in N_{i}} a_{i j}(t) \phi\left(y_{j}-y_{i}\right)+\rho_{i} \phi\left(y_{0}-y_{i}\right)\right)-L_{f_{i}} h_{i}}{L_{g_{i}} h_{i}},
$$

where $N_{i}=\left\{v_{j} \in v:\left(v_{i}, v_{j}\right) \in \varepsilon\right\}$ the set of neighbors of $v_{i}$, $a_{i j}(t)>0$ is the element of the time-varying adjacent matrix $A(t)$.

By substituting time varying cooperative control law (21) into system (12), we can get each subsystem's closed-loop dynamics:

$$
\begin{array}{r}
\dot{y}_{i}=c_{i}\left(\sum_{j \in N_{i}} a_{i j}(t) \phi\left(y_{j}-y_{i}\right)+\rho_{i} \phi\left(y_{0}-y_{i}\right)\right), \\
i=1,2, \ldots, n .
\end{array}
$$

Being similar to the fixed topology case, we can get the each auxiliary subsystem's state equation under the dynamic topology:

$$
\begin{array}{r}
\dot{\delta}_{i}=\dot{y}_{i}=-c_{i}\left(\sum_{j \in N_{i}} a_{i j}(t) \phi\left(\delta_{i}-\delta_{j}\right)+\rho_{i} \phi\left(\delta_{i}\right)\right) \\
i=1,2, \ldots, n .
\end{array}
$$

Assumption 8. The directed dynamic graph $G_{s(t)}$ for the dynamic communication topology is symmetric at each time $t$; that is, $a_{i j}(t)=a_{j i}(t), i, j=1,2, \ldots, n$.

\section{Charging System Cooperative Stability Analysis}

In this section, the cooperative stability analysis of the multicharging systems under fixed and dynamic topology with the aid of the Lyapunov function integrating LaSalle Invariant Principle is given. 
Lemma 9. Suppose $\xi_{i} \in R^{m}, \zeta_{j} \in R^{m}, \forall i, j=1,2, \ldots, n$ and $S=\left[s_{i j}\right]_{n \times n} \in R^{n \times n}$; if matrix $S$ is symmetric, then

$$
\begin{array}{r}
\frac{1}{2} \sum_{i=1}^{n} \sum_{j=1}^{n} s_{i j}\left(\xi_{i}-\xi_{j}\right)^{T} \phi\left(\zeta_{i}-\zeta_{j}\right) \\
=\sum_{i=1}^{n} \sum_{j=1}^{n} s_{i j} \xi_{i}^{T} \phi\left(\zeta_{i}-\zeta_{j}\right) .
\end{array}
$$

Proof. Consider

$$
\begin{aligned}
\frac{1}{2} \sum_{i=1}^{n} \sum_{j=1}^{n} s_{i j}\left(\xi_{i}-\xi_{j}\right)^{T} \phi\left(\zeta_{i}-\zeta_{j}\right) \\
=\frac{1}{2} \sum_{i=1}^{n} \sum_{j=1}^{n} s_{i j} \xi_{i}^{T} \phi\left(\zeta_{i}-\zeta_{j}\right) \\
-\frac{1}{2} \sum_{i=1}^{n} \sum_{j=1}^{n} s_{i j} \xi_{j}^{T} \phi\left(\zeta_{i}-\zeta_{j}\right) .
\end{aligned}
$$

Because the function $\phi(x)$ is a bounded odd function, the minus of the second term can be substituted into the brackets:

$$
\begin{aligned}
\frac{1}{2} \sum_{i=1}^{n} \sum_{j=1}^{n} s_{i j}\left(\xi_{i}-\xi_{j}\right)^{T} \phi\left(\zeta_{i}-\zeta_{j}\right) & \\
= & \frac{1}{2} \sum_{i=1}^{n} \sum_{j=1}^{n} s_{i j} \xi_{i}^{T} \phi\left(\zeta_{i}-\zeta_{j}\right) \\
& +\frac{1}{2} \sum_{i=1}^{n} \sum_{j=1}^{n} s_{i j} \xi_{j}^{T} \phi\left(\zeta_{j}-\zeta_{i}\right) .
\end{aligned}
$$

Then exchanging $i$ with $j$ in the second term, we get

$$
\begin{aligned}
\frac{1}{2} \sum_{i=1}^{n} \sum_{j=1}^{n} s_{i j}\left(\xi_{i}-\xi_{j}\right)^{T} \phi\left(\zeta_{i}-\zeta_{j}\right) \\
=\frac{1}{2} \sum_{i=1}^{n} \sum_{j=1}^{n} s_{i j} \xi_{i}^{T} \phi\left(\zeta_{i}-\zeta_{j}\right) \\
+\frac{1}{2} \sum_{i=1}^{n} \sum_{j=1}^{n} s_{j i} \xi_{i}^{T} \phi\left(\zeta_{i}-\zeta_{j}\right) .
\end{aligned}
$$

Since $S$ is symmetric, we obtain

$$
\begin{array}{r}
\frac{1}{2} \sum_{i=1}^{n} \sum_{j=1}^{n} s_{i j}\left(\xi_{i}-\xi_{j}\right)^{T} \phi\left(\zeta_{i}-\zeta_{j}\right) \\
=\sum_{i=1}^{n} \sum_{j=1}^{n} s_{i j} \xi_{i}^{T} \phi\left(\zeta_{i}-\zeta_{j}\right) .
\end{array}
$$

This completes the proof.

\subsection{Cooperative Stability Analysis under Fixed Topology}

Theorem 10. Consider the multicharging systems (5) with fixed communication topology. Under the Assumption 7 that the directed graph is symmetric and $\rho_{i}>0$ for at least one charger, the cooperative control objective below can be achieved by the control law (17):

$$
\lim _{t \rightarrow+\infty}\left|y_{i}(t)-y_{0}\right|=0 .
$$

That is, the output current of the parallel chargers can ultimately be consensus and track the desired reference current. Furthermore, the overall closed-loop system is asymptotically cooperative stable.

Proof. To prove the multicharging system's cooperative stability, we turn to analyze the auxiliary system's (20) stability indirectly.

For the auxiliary closed-loop system (20), the Lyapunov function candidate can be chosen as follows:

$$
V=\frac{1}{2} \sum_{i=1}^{n} \delta_{i}^{T} \delta_{i} .
$$

Differentiating the Lyapunov function $V$ along the auxiliary subsystem's trajectory with respect to time $t$ and then substituting the state equation of each subsystem into the derivative function $\dot{V}$, we can have

$$
\begin{aligned}
\dot{V} & =\sum_{i=1}^{n} \delta_{i}^{T} \dot{\delta}_{i} \\
& =\sum_{i=1}^{n} \delta_{i}^{T}\left(c_{i}\left(\sum_{j \in N_{i}} a_{i j} \phi\left(\delta_{j}-\delta_{i}\right)\right)-\rho_{i} \phi\left(\delta_{i}\right)\right) \\
& =-c_{i} \sum_{i=1}^{n} \delta_{i}^{T} \sum_{j \in N_{i}} a_{i j} \phi\left(\delta_{i}-\delta_{j}\right)-c_{i} \sum_{i=1}^{n} \delta_{i}^{T} \rho_{i} \phi\left(\delta_{i}\right) .
\end{aligned}
$$

According to the communication topology, we know $\sum_{j \in N_{i}} a_{i j}=\sum_{j=1}^{n} a_{i j}$.

The derivative function $\dot{V}$ (31) can be transformed into

$$
\begin{aligned}
\dot{V} & =-c_{i} \sum_{i=1}^{n} \delta_{i}^{T} \sum_{j=1}^{n} a_{i j} \phi\left(\delta_{i}-\delta_{j}\right)-c_{i} \sum_{i=1}^{n} \delta_{i}^{T} \rho_{i} \phi\left(\delta_{i}\right) \\
& =-c_{i} \sum_{i=1}^{n} \sum_{j=1}^{n} a_{i j} \delta_{i}^{T} \phi\left(\delta_{i}-\delta_{j}\right)-c_{i} \sum_{i=1}^{n} \delta_{i}^{T} \rho_{i} \phi\left(\delta_{i}\right) .
\end{aligned}
$$

Based on Lemma 9, the above equation (32) is equal to

$$
\begin{aligned}
\dot{V}= & -\frac{1}{2} c_{i} \sum_{i=1}^{n} \sum_{j=1}^{n} a_{i j}\left(\delta_{i}-\delta_{j}\right)^{T} \phi\left(\delta_{i}-\delta_{j}\right) \\
& -c_{i} \sum_{i=1}^{n} \delta_{i}^{T} \rho_{i} \phi\left(\delta_{i}\right) .
\end{aligned}
$$

Since $\phi\left(\delta_{i}-\delta_{j}\right)$ and $\left(\delta_{i}-\delta_{j}\right), \phi\left(\delta_{i}\right)$ and $\delta_{i}$ have the same sign componentwise, we get $\dot{V} \leq 0$. To this end, the overall auxiliary system is stable. 
Note that $\dot{V} \equiv 0$ implies that $\delta_{i}-\delta_{j}=0$ and $\delta_{i}=0$ when the associated directed graph is symmetric, which, in turn, implies that $\delta_{i}=\delta_{j}, \forall i \neq j$.

From LaSalle's Invariance principle, it follows that $\delta_{i} \rightarrow$ $\delta_{j}, \forall i \neq j$ asymptotically as $t \rightarrow+\infty$; that is, $\lim _{t \rightarrow+\infty} \mid \delta_{i}-$ $\delta_{j} \mid=0$ and $\lim _{t \rightarrow+\infty}\left|\delta_{i}\right|=0$. That is to say, the overall auxiliary closed-loop system is asymptotically cooperative stable.

Furthermore, we can obtain $\lim _{t \rightarrow+\infty}\left|y_{i}(t)-y_{0}\right|=0$, $\forall i \neq j$ since the fact is that $\delta_{i}=y_{i}-y_{0}$.

To this end, the current state of the all chargers can ultimately be consensus and track the desired reference current. Furthermore, the overall closed-loop system is asymptotically stable.

This completes the proof.

\subsection{Cooperative Stability Analysis under Dynamic Topology}

Theorem 11. Consider the multicharging systems (5) under dynamic communication topology. Under the Assumption 8 that the directed graph is symmetric at each time $t$ and $\rho_{i}>0$ for at least one charger, the current-sharing objective can be achieved under the proposed distributed time-varying cooperative control law (21).

That is to say, the output current of the all chargers can ultimately be consensus and track the desired reference current. Furthermore, the overall closed-loop system is asymptotically cooperative stable.

Proof. Being similar to the fixed topology case, the Lyapunov function candidate for the auxiliary system (23) can be chosen as follows:

$$
V=\frac{1}{2} \sum_{i=1}^{n} \delta_{i}^{T} \delta_{i}
$$

Differentiate the Lyapunov function $V$ along the auxiliary subsystem's trajectory with respect to time $t ; \dot{V}$ can be derived as follows:

$$
\dot{V}=-c_{i} \sum_{i=1}^{n}\left(\delta_{i}^{T} \sum_{j \in N_{i}} a_{i j}(t) \phi\left(\delta_{i}-\delta_{j}\right)+\rho_{i} \phi\left(\delta_{i}\right)\right) .
$$

However, the time varying adjacent matrix $A(t)$ of the direct graph is symmetric at each time $t$; that is, $a_{i j}(t)=a_{j i}(t)$.

By virtue of Lemma 9, $\dot{V}$ can be transformed to be

$$
\begin{aligned}
\dot{V}= & -\frac{1}{2} c_{i} \sum_{i=1}^{n} \sum_{j=1}^{n} a_{i j}(t)\left(\delta_{i}-\delta_{j}\right)^{T} \phi\left(\delta_{i}-\delta_{j}\right) \\
& -c_{i} \sum_{i=1}^{n} \delta_{i}^{T} \rho_{i} \phi\left(\delta_{i}\right) .
\end{aligned}
$$

Similar to Theorem 10, the function $y=\phi(x)$ is monotonically increasing. $\phi(x) \geq 0$ when $x \geq 0$, and $\phi(x) \leq$ 0 when $x \leq 0$. That is to say, $\phi(x)$ and $x$ have the same sign componentwise. And then, we can obtain that $\dot{V} \leq 0$. Consequently, the overall auxiliary systems is stable.
TABLE 1: The main parameters for the charging system model.

\begin{tabular}{lccc}
\hline Charger $i$ & $L_{i}(\mathrm{mH})$ & $r_{i}(\Omega)$ & $U_{d_{i}}(\mathrm{~V})$ \\
\hline 1 & 3.05 & 0.0035 & 1335 \\
2 & 3.12 & 0.0031 & 1272 \\
3 & 2.95 & 0.0029 & 1295 \\
4 & 3.01 & 0.0040 & 1371 \\
\hline
\end{tabular}

TABLE 2: The simulation parameters for the super capacitor.

\begin{tabular}{lccc}
\hline Parameter & $R_{1}$ & $C_{0}$ & $C_{v}$ \\
\hline Value & $5.6 \mathrm{~m} \Omega$ & $92.3 \mathrm{~F}$ & $U_{c} * 0.0747 \mathrm{~F} / \mathrm{V}$ \\
\hline
\end{tabular}

Similarly to the proof of Theorem 10 with the help of LaSalle's Invariance principle, the overall auxiliary closeloop system is asymptotically stable provided that the direct dynamical communication topology is symmetric at each time interval. Furthermore, the cooperative objective can be achieved while the overall closed-loop system is asymptotically cooperative stable under the dynamic topology.

This completes the proof.

\section{Case Studies}

In this section, we use the charging test system shown in Figure 2 to validate the feasibility of the proposed cooperative control scheme. Three different cases below are considered and compared in this section.

Case A. The charging current is chosen only to be $450 \mathrm{~A}$ in the whole charging process.

Case $B$. The whole charging process includes two sequential phases, namely, a fast charging phase and a trickle charging phase. At the fast charging phase, the charging current is chosen to be $450 \mathrm{~A}$. At the trickle charging phase, the charging current is chosen to be $100 \mathrm{~A}$.

Case C. On the basis of Case B, the coupling strength in the cooperative current-sharing controller can be tuned to be much larger in order to improve the response speed.

The main parameters for the charging systems model are given in Table 1. The simulation parameters for the supercapacitor are shown in Table 2. The initial current of the chargers is $0 \mathrm{~A}$. In Case $\mathrm{B}$, the supercapacitor's initial voltage $U_{c}$ is $500 \mathrm{~V}$, nominal voltage is $900 \mathrm{~V}$, and nominal charging current is $450 \mathrm{~A}$ and $100 \mathrm{~A}$, which means that the supercapacitor's residual voltage is $500 \mathrm{~V}$ when the energy storage type light rail vehicle reaches the platform and the supercapacitor should be charged to be about $870 \mathrm{~V}$ by 4 chargers in parallel with each other by choosing a $450 \mathrm{~A}$ charging current in a very short period of time, and then the charging current is turned to be $100 \mathrm{~A}$, which charges the supercapacitor to $900 \mathrm{~V}$.

The given communication topology between each charging subsystem is shown in Figure 6. 


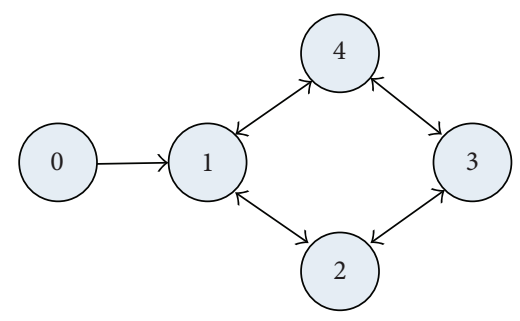

Figure 6: The given communication topology between each charging subsystem.

The specific final controller in the simulation is formulated below:

$$
\begin{aligned}
u_{i}= & \left(c_{i}\left(\sum_{j \in N_{i}} a_{i j} \phi\left(y_{j}-y_{i}\right)+\rho_{i} \phi\left(y_{0}-y_{i}\right)\right)\right. \\
& \left.-\left(-\frac{r_{i}}{L_{i}} y_{i}-\frac{U_{c}}{L_{i}}\right)\right) L_{i} \\
= & c_{i}\left(\sum_{j \in N_{i}} a_{i j} \phi\left(y_{j}-y_{i}\right)+\rho_{i} \phi\left(y_{0}-y_{i}\right)\right) L_{i}+r_{i} y_{i}+U_{c},
\end{aligned}
$$

where $r_{i}, L_{i}$ are given in Table $1, \rho_{1}=1$, and $\rho_{2}=\rho_{3}=\rho_{4}=0$. In Case $\mathrm{A}$ and Case $\mathrm{B}$, the coupling strengths are $c_{1}=2.25$, $c_{2}=2.5, c_{3}=2.75$, and $c_{4}=3$, while $c_{1}=4.25, c_{2}=4.5$, $c_{3}=4.75$, and $c_{4}=5$ in Case C. In Case A, the current-sharing object $y_{0}=450 \mathrm{~A}$. In Case B and Case C, the current-sharing object $y_{0}=450 \mathrm{~A}$ in the fast charging phase and $y_{0}=100 \mathrm{~A}$ in the trickle charging phase. The initial current $y_{i}(0)=0 \mathrm{~A}$, $i=1,2,3,4$, the supercapacitor's initial voltage $U_{c}(0)=500 \mathrm{~V}$ in all Cases. The entry $a_{i j}$ of the adjacency matrix $A$ for the given communication topology in Figure 6 is

$$
A=\left[\begin{array}{cccc}
0 & 1.25 & 0 & 1.25 \\
1.25 & 0 & 1.5 & 0 \\
0 & 1.5 & 0 & 1.75 \\
1.25 & 0 & 1.75 & 0
\end{array}\right]
$$

The specific function expression for the saturation function in Figures 7-11 is given as follows:

$$
\phi(x)= \begin{cases}a, & x \geq a \\ x, & x \in[-a, a] \\ -a, & x \leq-a,\end{cases}
$$

where $a$ is parameter which can character the upper and lower condition of the saturation function. Here, $a=300$ in the simulation of Figures 7-11.

When the coupling strength $c_{i}$ is chosen to be $c_{1}=2.25$, $c_{2}=2.5, c_{3}=2.75$, and $c_{4}=3$, the current-sharing curve for Case A under the communication topology in Figure 6 is plotted in Figure 7, which illustrates that the current has been balanced and the consensus and cooperative objective have been accomplished.

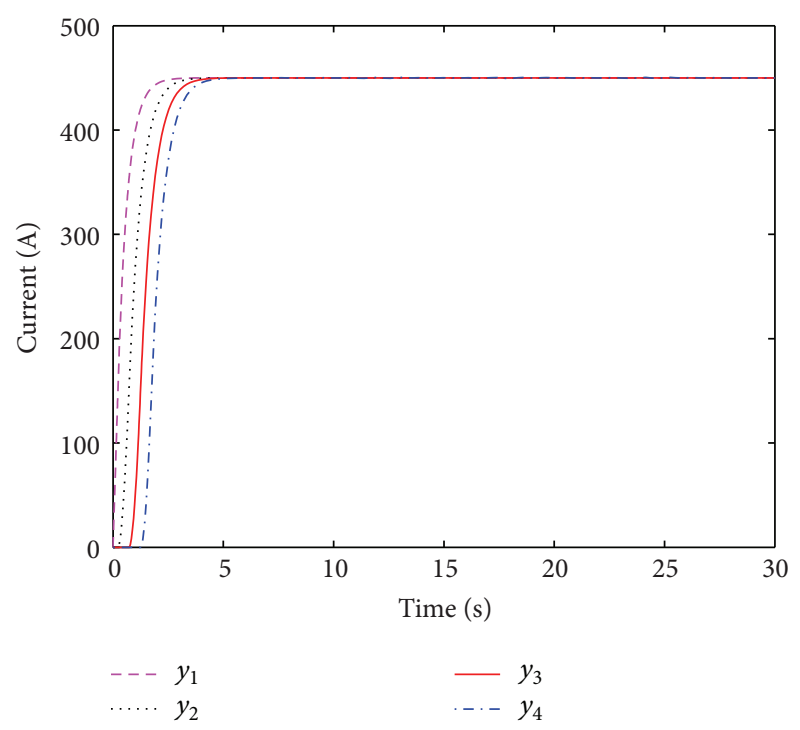

FIGURE 7: The current-sharing curve for Case A under the given communication topology.

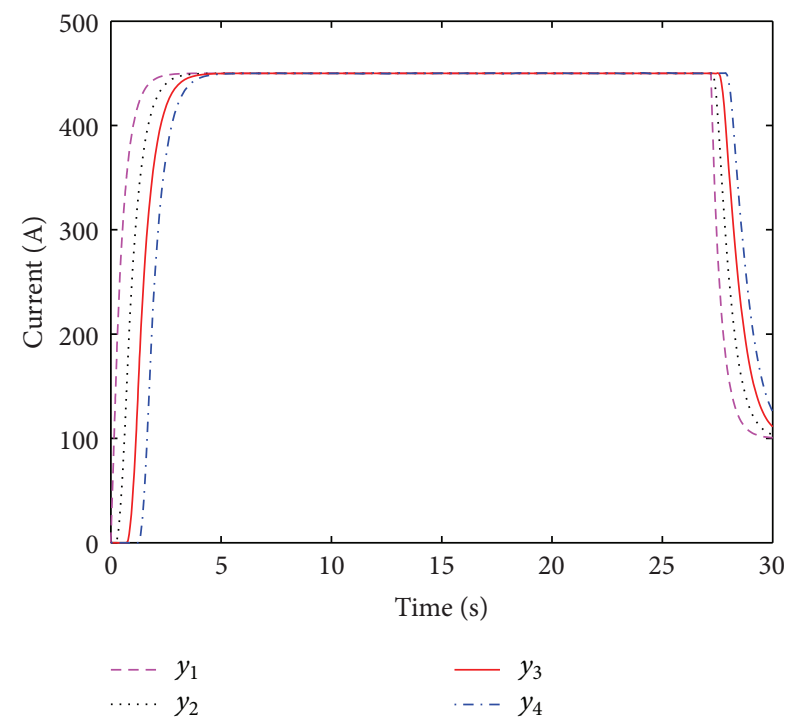

Figure 8: The current-sharing curve for Case B under the given communication topology.

As shown in Figure 8 for Case B, the charging current ascends to be $450 \mathrm{~A}$ from $0 \mathrm{~A}$ within $4 \mathrm{~s}$, and then it goes to be constant current charging stage until the supercapacitor is charged to be about $870 \mathrm{~V}$. Since the supercapacitor's voltage will decline at the end of charging stage, as shown in Figure 9(a), if we always choose the large charging current in the whole charging process as Case $\mathrm{A}$, the charging current in Case B is then reduced to $100 \mathrm{~A}$ at the end of charging stage. Such a stage is called the trickle charging phase. As Figure 9(b) shows, the voltage is charged fully from $500 \mathrm{~V}$ to about $900 \mathrm{~V}$ in less than 30 second, illustrating that voltage objective has been achieved by adopting the proposed control strategy in Case B. 


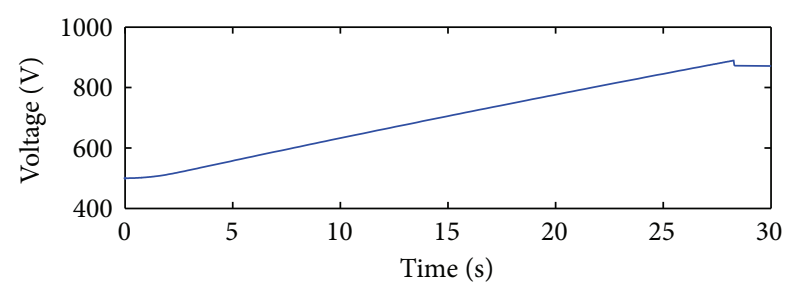

- Case A

(a)

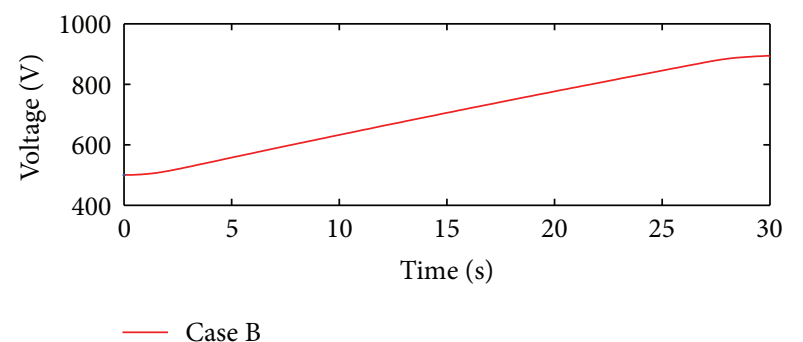

(b)

FIGURE 9: The charging voltage curve of the whole charging system under the given communication topology, (a) Case A and (b) Case B.

Comparing Figure 9(a) with Figure 9(b), the supercapacitor's voltage will decline about $18 \mathrm{~V}$ in Case $\mathrm{A}$ as shown in Figure 9(a) if the charging current is chosen only to be $450 \mathrm{~A}$. On the contrary, if we choose $450 \mathrm{~A}$ as the charging current at the first stage and $100 \mathrm{~A}$ at the last stage in Case B, the supercapacitor can be charged fully to be $900 \mathrm{~V}$ as shown in Figure 9(b).

The current-sharing coefficient curves in Case B and Case $\mathrm{C}$ under the proposed cooperative control scheme are plotted in Figure 11, which is used to measure the balance of the current. When the coupling strengths are chosen to be larger as in Case C, the current-sharing coefficient converges to be 1 as shown in Figure 11(b), which is much better than that of Case B as shown in Figure 11(a).

Comparing Case B with Case $\mathrm{C}$, which are shown in Figures 8 and 10, the convergence performance will be better if the coupling strengths are chosen to be larger. In a word, we can conclude that if we choose a larger coupling strength, the convergence performance will be improved, which will unfortunately result in a larger ripple peak value at the same time.

Next, simulation comparison between the proposed cooperative current-sharing control and the proportional integral (PI) control is given below. Without loss of generality, we consider the most basic charging scene, Case $\mathrm{A}$. The initial currents are $y_{1}(0)=50 \mathrm{~A}, y_{2}(0)=45 \mathrm{~A}, y_{3}(0)=60 \mathrm{~A}$, and $y_{4}(0)=55 \mathrm{~A}$ and the supercapacitor's initial voltage is $U_{c}(0)=500 \mathrm{~V}$ in such two schemes.

The charging curve for Case $\mathrm{A}$ is given in Figure 12 by choosing PI control law. The proportional control gains $k_{p}=$ 0.2 and the integral control gains $k_{i}=0.13$, which are the same for all chargers. Although the control objects are known

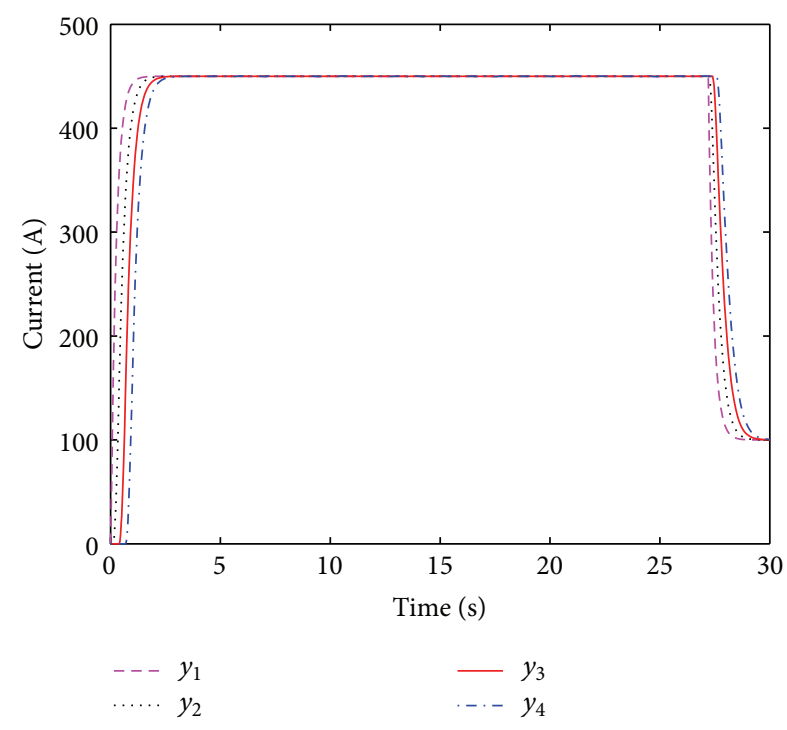

FIgure 10: The current-sharing curve for Case $\mathrm{C}$ under the given communication topology with coupling strengths $c_{1}=4.25, c_{2}=4.5$, $c_{3}=4.75$, and $c_{4}=5$.

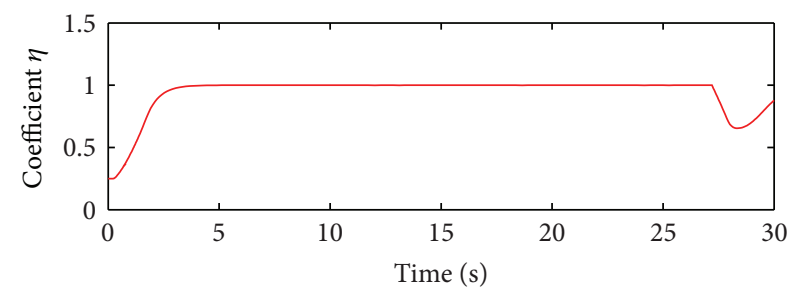

- Case B

(a)

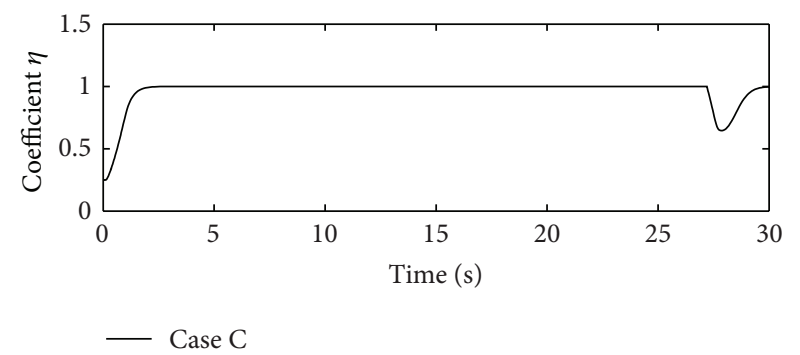

(b)

FIGURE 11: The current-sharing coefficient curve under the proposed cooperative control scheme with different coupling strengths in (a) Case $\mathrm{B}, c_{1}=2.25, c_{2}=2.5, c_{3}=2.75$, and $c_{4}=3$ and (b) Case $\mathrm{C}$, $c_{1}=4.25, c_{2}=4.5, c_{3}=4.75$, and $c_{4}=5$.

to each charger, they do not interact with each other. The current-sharing result is not well sometimes as shown in Figure 12. In addition, there exist overshoot and static error by using the PI current-sharing control approach. Too large overshoot and static error are not allowed in the charging system. The initial charging current will be very large if the existing overshoot is too large. Not only the supercapacitor 


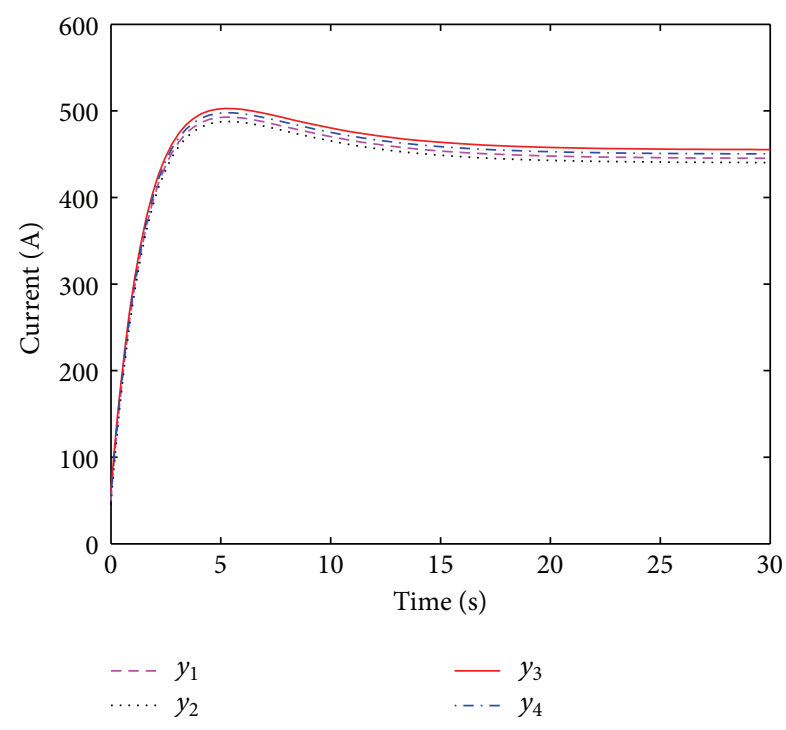

Figure 12: The current-sharing curve for Case A under the PI control law.

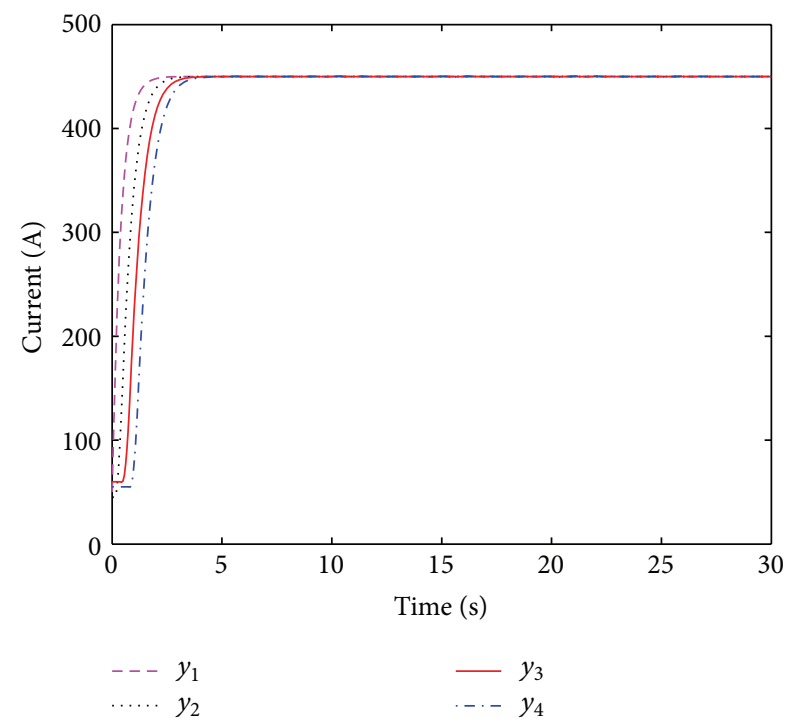

FIgURE 13: The current-sharing curve for Case A under the cooperative control law.

and charger will be damaged, but also the operator may be endangered.

But in the proposed cooperative control scheme, each charger can communicate with each other through the network and the control objective is just needed to be preconfigured in one charger. The current-sharing result is shown in Figure 13 when the initial current is not zero, where $c_{1}=2.65, c_{2}=2.9, c_{3}=3.15$, and $c_{4}=3.4$. There is not any overshoot and static error by using the proposed cooperative current-sharing approach compared with PI control method within the same rising time $t_{r}$.

\section{Conclusions}

In this paper, the distributed cooperative control of the nonlinear and nonidentical multiagent systems is adopted to carry out current-sharing strategy for charging system of energy storage type light rail vehicle, that addresses the current balance problem. Input-output feedback linearization is introduced to convert the distributed cooperative current-sharing control of multicharging with nonidentical subsystem to a first-order integrator consensus problem. The cooperative current-sharing control law is put forward by introducing a general saturation function. The proposed cooperative current-sharing strategy is distributed; each distributed charging subsystem only needs its own information and the information of some neighbors. The whole closedloop charging system is proved to be cooperative stable when the directed graph is symmetric. The nonidentical feature of the charging system can be removed by the proposed control law, thus ensuring that the overall system is stable and the cooperative current-sharing objective can be achieved. The response speed can be improved by tuning current-sharing control parameters. Case studies show that our currentsharing approach is effective and feasible.

\section{Conflict of Interests}

The authors declare that there is no conflict of interests regarding the publication of this paper.

\section{Acknowledgment}

This work was supported by the National Natural Science Foundation of China (nos. 61071096, 61003233 and 61073103) and Specialized Research Fund for the Doctoral Program of Higher Education (nos. 20100162110012 and 20110162110042 ).

\section{References}

[1] W. Chen, X. Ruan, H. Yan, and C. K. Tse, "DC/DC conversion systems consisting of multiple converter modules: stability, control, and experimental verifications," IEEE Transactions on Power Electronics, vol. 24, no. 6, pp. 1463-1474, 2009.

[2] B. K. De, B. Bolsens, J. van den Keybus, A. Woyte, J. Driesen, and R. Belmans, "A voltage and frequency droop control method for parallel inverters," IEEE Transactions on Power Electronics, vol. 22, no. 4, pp. 1107-1115, 2007.

[3] J. B. Wang, "Study of cable resistance and remote-sensing scheme in parallel DC/DC converter system via primary droop current-sharing control," IET on Power Electronics, vol. 5, no. 6, pp. 885-898, 2012.

[4] S. K. Mazumder, M. Tahir, and K. Acharya, "Master-slave current-sharing control of a parallel DC-DC converter system over an RF communication interface," IEEE Transactions on Industrial Electronics, vol. 55, no. 1, pp. 59-66, 2008.

[5] P. Li and B. Lehman, "A design method for paralleling current mode controlled DC-DC converters," IEEE Transactions on Power Electronics, vol. 19, no. 3, pp. 748-756, 2004.

[6] Y. Cao, W. Yu, W. Ren, and G. Chen, "An overview of recent progress in the study of distributed multi-agent coordination," 
IEEE Transactions on Industrial Informatics, vol. 9, no. 1, pp. 427-438, 2013.

[7] R. Olfati-Saber, J. A. Fax, and R. M. Murray, "Consensus and cooperation in networked multi-agent systems," Proceedings of the IEEE, vol. 95, no. 1, pp. 215-233, 2007.

[8] W. Ren, R. W. Beard, and E. M. Atkins, "Information consensus in multivehicle cooperative control," IEEE Control Systems Magazine, vol. 27, no. 2, pp. 71-82, 2007.

[9] G. Antonelli, "Interconnected dynamic systems: an overview on distributed control," IEEE Control Systems Magazine, vol. 33, no. 1, pp. 76-88, 2013.

[10] Z. Qu, J. Wang, and R. A. Hull, "Cooperative control of dynamical systems with application to autonomous vehicles," IEEE Transactions on Automatic Control, vol. 53, no. 4, pp. 894911, 2008.

[11] H. Xin, Z. Qu, J. Seuss, and A. Maknouninejad, "A selforganizing strategy for power flow control of photovoltaic generators in a distribution network," IEEE Transactions on Power Systems, vol. 26, no. 3, pp. 1462-1473, 2011.

[12] A. Bidram, A. Davoudi, F. L. Lewis, and J. M. Guerrero, "Distributed cooperative secondary control of microgrids using feedback linearization," IEEE Transactions on Power System, vol. 28, no. 3, pp. 3462-3470, 2013.

[13] R. Diestel, Graph Theory, vol. 173 of Graduate Texts in Mathematics, Springer, New York, NY, USA, 1997.

[14] W. Ren, "Consensus tracking under directed interaction topologies: algorithms and experiments," IEEE Transactions on Control Systems Technology, vol. 18, no. 1, pp. 230-237, 2010.

[15] H. Zhang, F. L. Lewis, and Z. Qu, "Lyapunov, adaptive, and optimal design techniques for cooperative systems on directed communication graphs," IEEE Transactions on Industrial Electronics, vol. 59, no. 7, pp. 3026-3041, 2012. 


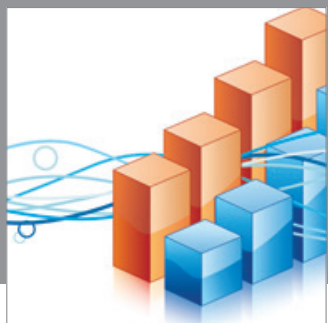

Advances in

Operations Research

mansans

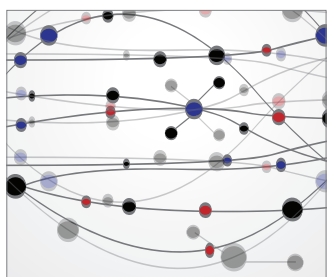

The Scientific World Journal
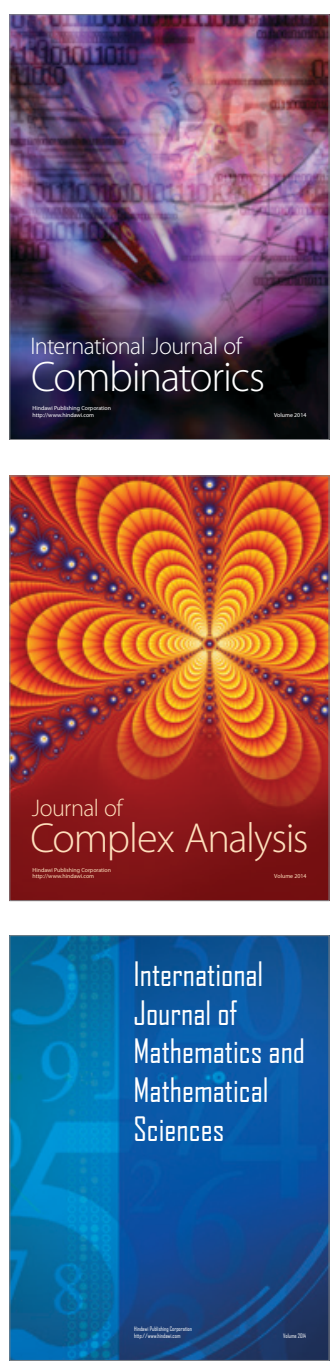
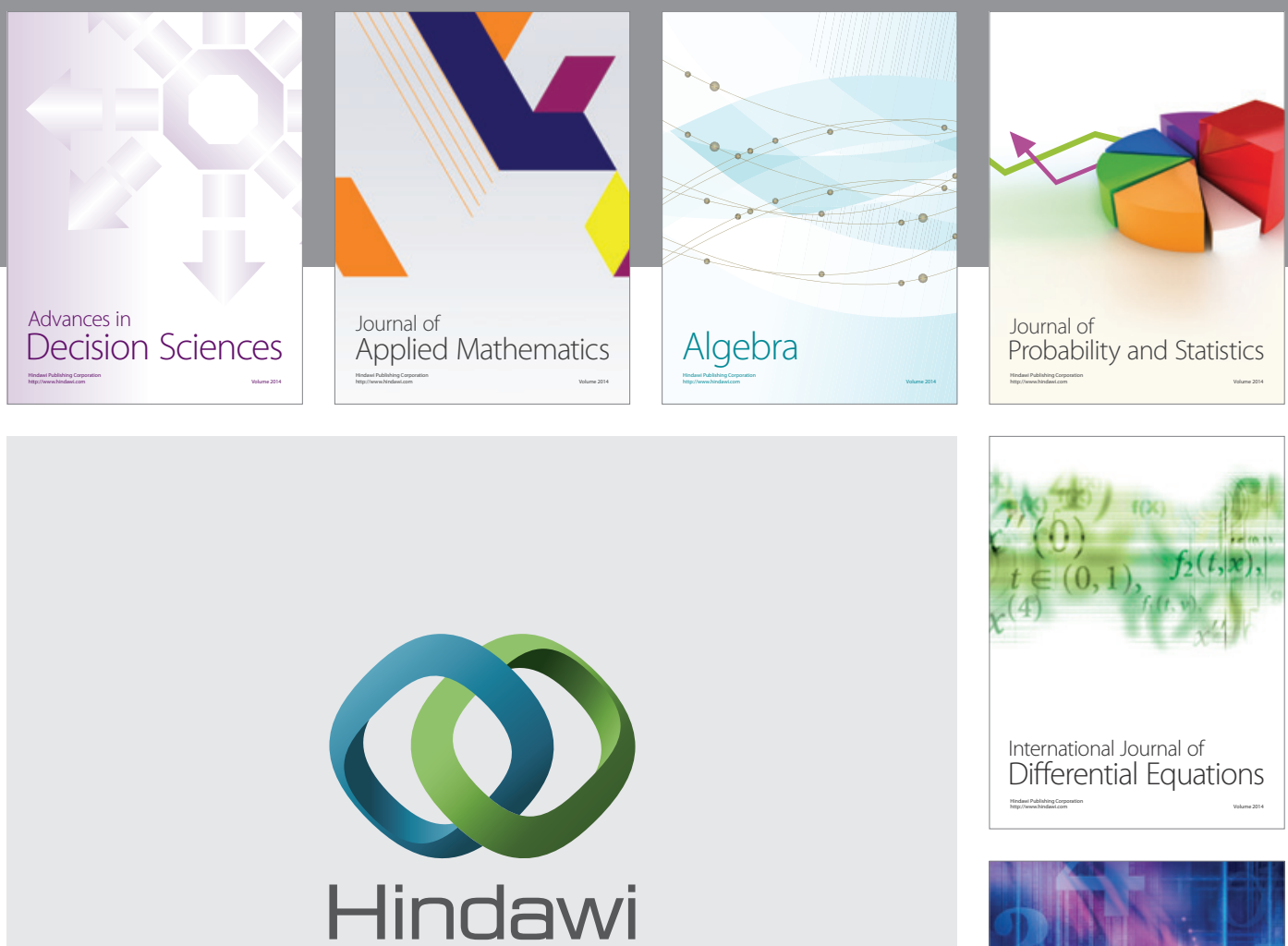

Submit your manuscripts at http://www.hindawi.com
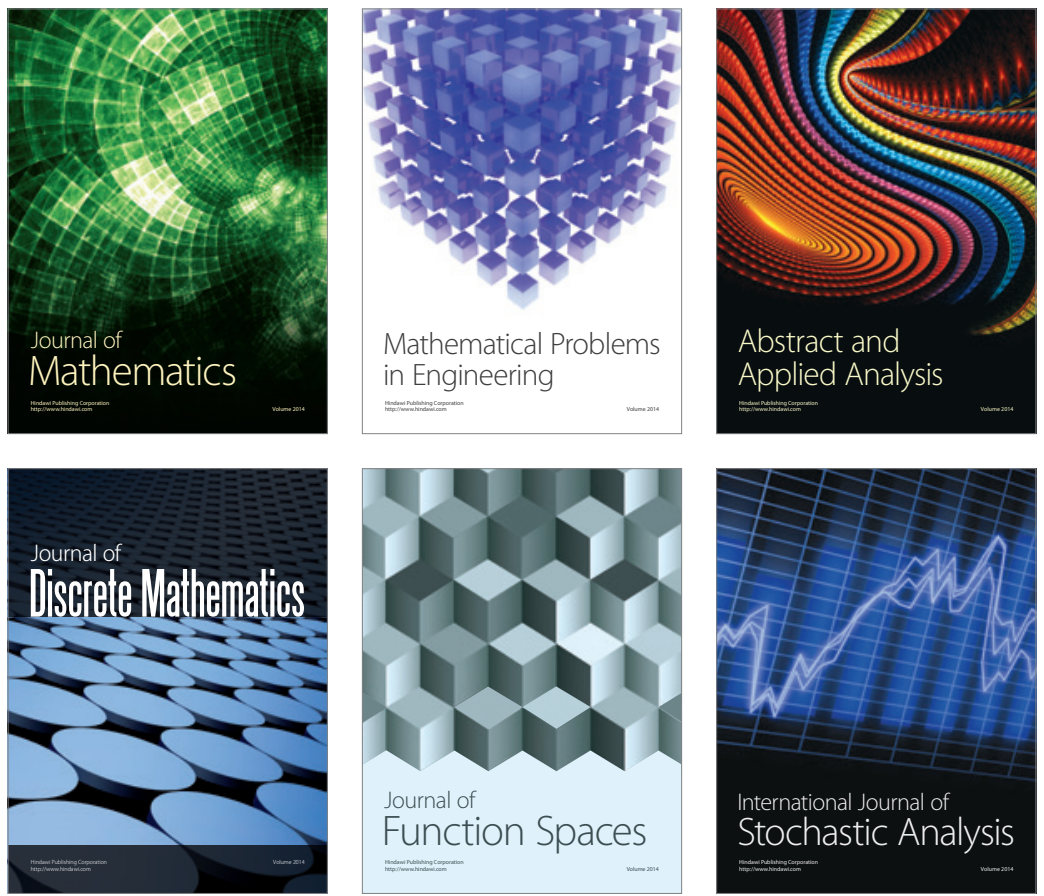

Journal of

Function Spaces

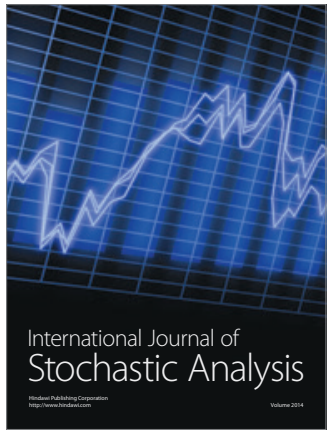

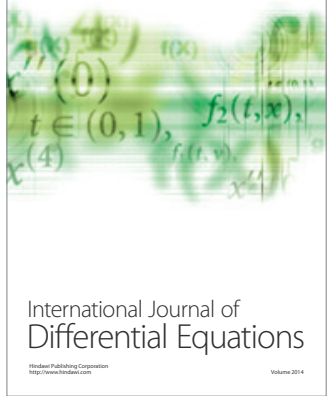
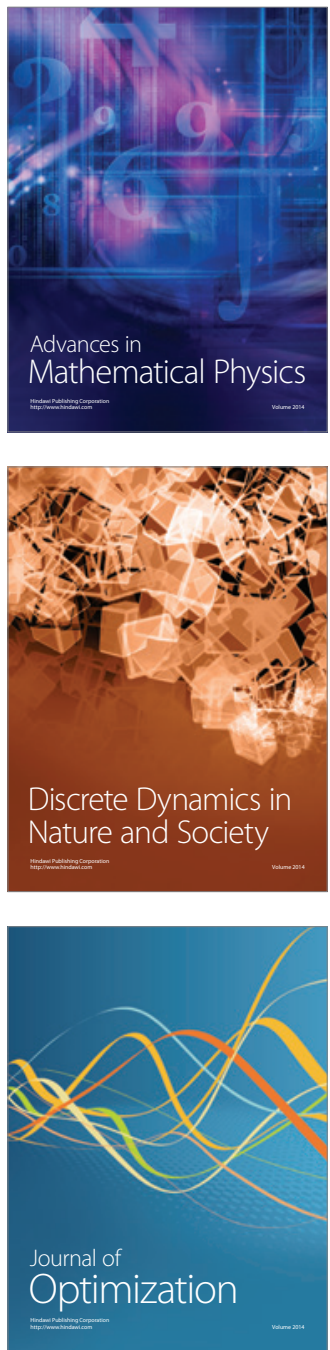